Julia C. BULLOcK, Ayako KANO \& James WELKER (eds), Rethinking Japanese Feminisms

Honolulu, University of Hawai'i Press, 2018

\title{
Christine Lévy
}

\section{(2) OpenEdition}

Journals

\section{Édition électronique}

URL : https://journals.openedition.org/clio/16797

DOI : 10.4000/clio. 16797

ISSN : 1777-5299

\section{Éditeur}

Belin

Édition imprimée

Date de publication : 1 juillet 2019

Pagination : $317-320$

ISSN : 1252-7017

\section{Référence électronique}

Christine Lévy, « Julia C. вuLlock, Ayako kano \& James welker (eds), Rethinking Japanese Feminisms », Clio. Femmes, Genre, Histoire [En ligne], 49 | 2019, mis en ligne le 01 juillet 2019, consulté le 04 janvier 2023. URL : http://journals.openedition.org/clio/16797 ; DOI : https://doi.org/10.4000/clio.16797

Ce document a été généré automatiquement le 4 janvier 2023.

Tous droits réservés 


\section{Julia C. BULLOCK, Ayako KANO \& James WELKER (eds), Rethinking Japanese Feminisms}

Honolulu, University of Hawai'i Press, 2018

Christine Lévy

\section{RÉFÉRENCE}

Julia C. BULlock, Ayako KANO \& James WELKER (eds), Rethinking Japanese Feminisms, Honolulu, University of Hawai'i Press, 2018, 201 p.

1 Comme son titre l'indique, cet ouvrage collectif renouvelle réflexions et analyses autour des mouvements et figures féministes du Japon dans différents domaines. L'introduction nous donne un aperçu historique et synthétique très utile pour resituer les chapitres qui suivent. Elle souligne l'apparition de figures féministes (Kishida Toshiko, Fukuda Hideko) dès le début de la construction de l'État moderne japonais. Leur combat pour accéder à l'espace politique resta minoritaire alors que le principe de la « bonne épouse, mère avisée » devenait le mot d'ordre central définissant la place de la femme dans la famille. La revue mensuelle Seitô (1911-1916) deviendra le lieu d'où sortiront de nombreuses militantes féministes de premier plan, dont Yamakawa Kikue (1890-1980). Elyssa Faison montre dans le premier chapitre que l'hostilité de Yamakawa vis-à-vis du « féminisme bourgeois » ne l'avait pas empêchée de revendiquer le suffrage féminin dès avant 1946. Son originalité, par rapport à la Ligue pour le suffrage féminin, tenait à l'intégration aussi des colonisé.es dans la revendication du suffrage universel et de l'égalité des salaires. Elle explique son ralliement au camp démocratique pour former le Club Démocrate des Femmes (Fujin Minshu Kurabu) en mars 1946 par la conscience du rôle qu'elle pouvait jouer dans le nouveau contexte politique. Elle apparait la personnalité la mieux placée pour proposer un agenda politique en faveur des femmes dans le cadre du gouvernement de coalition socialiste de Katayama Tetsu 
(1947-1948). La continuité de son engagement féministe avant et après-guerre est bien mise en valeur dans cet article.

2 La première partie est ainsi constituée d'un ensemble d'articles qui discutent le rôle de certaines figures historiques importantes ou d'organisations considérées comme marginales y compris dans le mouvement féministe, tels les premiers groupes de lesbiennes dans les années 1970 (James Welker).

3 Le second chapitre, très novateur, d'Hillary Maxson, porte sur le rôle du Premier Congrès des mères (Hahaoya taikai) qui fut à l'initiative de la tenue, en juillet 1955, du Congrès mondial des mères en Suisse. Cette mobilisation naquit de la protestation contre les essais nucléaires menés par les Américains dans les atolls Bikini, qui avaient entraîné, le $1^{\mathrm{er}}$ mars 1954 , l'irradiation de tout le personnel navigant du thonier japonais Daigo Fukuryûmaru et la mort de son opérateur radio pour irradiation aiguë moins de sept mois plus tard.

Hillary Maxson propose la notion de "féminisme matricentrique ", en se référant à l'ouvrage de Andrea O'Reilly, The $21^{\text {st }}$ Century Motherhood Movement, pour l'opposer à la " maternité martiale » imposée aux mères pendant la période militaire. La description des liens directs entre Hiratsuka Raichô (fondatrice de la revue Seitô) et Eugénie Cotton pour la convocation du Congrès mondial des mères est très instructive. Le but de ce Congrès était de transformer la maternité en une force politique publique déployée par les femmes elles-mêmes pour exiger de l'État, protection et aide dans l'éducation des enfants. L'une des treize résolutions appelle les mères à ne plus faire de distinction entre filles et garçons, et si le Congrès mondial resta éphémère, le Congrès des mères au Japon continua à se réunir annuellement. Il joua également un rôle important dans le grand mouvement (Anpo tôsô) contre le renouvellement du traité de sécurité nippoaméricain, en 1960. Par ailleurs le Congrès mondial des mères est le moment où, pour la première fois, les féministes japonaises furent à l'initiative d'un mouvement international.

5 La seconde partie, dédiée à l'éducation et à l'emploi, nous fait découvrir des aspects peu connus de l'histoire des femmes et des féminismes. Le chapitre que Julia C. Bullock consacre à une éducatrice féministe Koizumi Ikuko (1892-1964) - qui s'est battue très tôt pour la coéducation des filles et garçons (Danjo kyôgakuron) dans les écoles, bien avant son adoption par la Loi fondamentale sur l'Éducation de 1947 - offre une nouvelle perspective sur l'histoire des mouvements éducatifs. Koizumi Ikuko mit l'individu au centre de sa conception éducative afin de dépasser l'opposition entre égalité et différence sexuelles et elle lutta contre la réduction de toutes les femmes à un stéréotype. Pour elle, l'individu est un facteur qui doit primer sur le genre. Ses propositions de coéducation, adoptées par la Chambre basse en 1933 mais rejetées par la Chambre des Pairs, montre qu'elle faisait partie d'un courant libéral significatif de l'époque.

6 La réflexion que mène Nancy Stalker sur la prospérité de l'enseignement de l'ikebana (arrangement floral) dans les années 1960 est également inédite. Cette pratique, enracinée dans la tradition japonaise et qui a eu le succès que l'on sait en Occident, eut l'avantage d'offrir des opportunités professionnelles tout à fait uniques aux femmes au foyer pendant la Haute croissance (1955-1973). Loin de l'image genrée de cette pratique, à l'époque Edo (1603-1868), seul $1 \%$ des adeptes de la cérémonie de thé était constitué de femmes, et les écoles d'ikebana leur étaient fermées. Le boom du recrutement de professeures femmes dans cet art, dans les années 1960, est donc un 
phénomène récent. On a là un cas paradoxal où, tout en soutenant l'idéologie de la bonne épouse et mère avisée, un secteur a offert des opportunités inexistantes par ailleurs. Pour les femmes seules, pour les divorcées, c'est l'industrie du tourisme avec ses auberges japonaises (ryokan) qui leur a ouvert des débouchés, tant comme patronnes que comme employées. Chris McMorran s'interroge sur les effets d'un tel métier où les normes féminines constituent des performances rémunérées. Son enquête montre que la satisfaction des travailleuses de ce secteur vient de leur indépendance vis-à-vis d'un homme.

7 À travers ses articles nombreux et variés, la question que pose cet ouvrage est aussi celui de l'héritage féministe spécifique au Japon : il n'est pas l'apanage des mouvements féministes et le rôle des artistes et auteures est interrogé dans la troisième partie. En quoi les créatrices ou leurs créations présenteraient-elles un caractère féministe? On peut lire leurs œuvres comme des réponses à la misogynie de la société, à la violence masculine, comme une confrontation à leur rôle sexuel et reproductif. C'est ainsi que l'œuvre du dessinateur Takabatake Kashô (1888-1966) est qualifiée de féminisme de l'ambiguïté, quand elle met en valeur des corps hermaphrodites et pose un regard distancé sur un monde sexuel binaire.

8 Barbara Hartley propose de valoriser d'un point de vue féministe les études d'auteures aussi différentes qu'Ariyoshi Sawako et Sono Ayako; quant à Kirino Natsuo qui, dans ses romans policiers, dissèque la misogynie intériorisée par les femmes, elle montre selon Kathryn Hemman comment la femme est cruellement dévalorisée dans la société.

Les frontières et leurs porosités sont aussi repensées dans la quatrième partie, cette fois sur le plan géographique, avec à nouveau un article sur Yamakawa Kikue, traductrice des écrits d'Edward Carpenter sur l'homosexualité. Setsu Shigematsu interroge les limites et les contradictions internes au ûman ribu (c'est à dire lu mouvement de libération des femmes, né avec la première manifestation le 21 octobre 1970 à Tokyo) par rapport à la question de l'ethnicité et du lesbianisme. Akwi Seo présente le rôle du réseau Yeoseong-Net dans la concrétisation de la solidarité entre féministes japonaises et coréennes sur la question du soutien aux anciennes "femmes de réconfort », ces esclaves sexuelles de l'armée impériale japonaise durant la guerre d'Asie-Pacifique (1931-1945). Et J. Keith Vincent nous fait découvrir le rôle clé joué par Takemura Kazuko (1954-2011), traductrice entre autres de Judith Butler et Gayatri Spivak, dans les échanges nippo-américains visant à combattre la misogynie et l'hétéronormativité au Japon.

10 Dans cette seconde décennie du troisième millénaire, le statut des femmes dans la société japonaise reste objet de controverses et, à l'heure où le Premier ministre Abe Shinzô qui s'était illustré par son antiféminisme dans les années 2000 prône un "Japon où brillent les femmes » avec les Abenomics (politique économique promue Abe Shinzô), ces réflexions permettent de renouveler les débats autour de la définition des féminismes et de mesurer leur ancrage dans la société japonaise. 


\section{AUTEURS}

\section{CHRISTINE LÉVY}

Université Bordeaux Montaigne

CRCAO 